\title{
14. Spotlight: Das „Bürgerdelphi Keimbahntherapie“
}

\subsection{Ausgangslage: Keimbahntherapie und Bürger*innenbeteiligung}

Im November 2018 teilte der chinesische Biophysiker He Jiankui der Nachrichtenagentur Associated Press (AP) mit, das Genom zweier Zwillingsmädchen verändert zu haben (siehe auch Fehse et al., Kap. 9). Sein Ziel sei es gewesen, die Zellen der Kinder resistent gegen HI-Viren werden zu lassen. Diese Äußerung wirkte wie eine „CRISPR bombshell“ (Normile, 2018), denn der Forscher hatte die CRISPR/Cas9-Technik ${ }^{1}$ an menschlichen Keimzellen verwendet und die veränderten Embryonen in eine menschliche Gebärmutter eingepflanzt - und damit gezeigt, wie Forschung legale und ethische Grenzen überschreiten kann. Deren Sicherung wurde dann auch umgehend eingefordert, u. a. von einer der Co-Erfinderinnen von CRISPR, Jennifer Doudna (Berkeley News, 2018; siehe auch Fehse et al., Kap. 9). Die mediale Resonanz auf diesen Einsatz der „Genschere“ war international hoch. Der Deutsche Ethikrat verurteilte Hes Vorgehen und attestierte ihm in einer Pressemitteilung eine ernste ethische Pflichtverletzung (Deutscher Ethikrat, 2018). 2019 legte dieser eine Stellungnahme vor, in der er Keimbahneingriffe als unverantwortlich einschätzte und sich für verbindliche internationale Vereinbarungen aussprach (Deutscher Ethikrat, 2019).

Hes „Bombe“ platzte kurz nach Abschluss unseres explorativen „Bürgerdelphi Keimbahntherapie“ (BUEDEKA), ${ }^{2}$ einem Deliberationsformat, d. h. einem Instrument zur Meinungs- und Entscheidungsfindung unter Einbeziehung von zufällig gewählten Bürger*innen. Es wurde von Ralf Grötker (Explorat Berlin; Grötker, 2017) entwickelt

1 Die CRISPR/Cas9-Technik dient dem gezielten Eingriff in das Erbgut von Organismen und wird oft auch als „Genschere“ bezeichnet. Zur Erläuterung dieser Technik und ihrer Anwendungspotenziale siehe Clemens, Kap. 7, und Fehse et al., Kap. 9.

2 Eine ausführliche Dokumentation des Projekts findet sich unter: www.buedeka.de [16.06.2021]. 
und von ihm in Kooperation mit meinem Lehrstuhl umgesetzt; gefördert vom Bundesministerium für Bildung und Forschung (BMBF).

CRISPR/Cas9 und die Keimbahntherapie bieten sich für ein Bürger*innenbeteiligungsverfahren an, weil sie massive Auswirkungen auf die Alltagswelt haben können etwa, weil durch dieses Verfahren auch verbessernde Eingriffe in den menschlichen Körper denkbar werden („Enhancement“). Ethisch fundierte Regeln des Zusammenlebens und des Forschens werden notwendig, die es zu entwickeln gilt. Unter der Annahme, dass es die Demokratie stärkt, wenn Bürger*innen mit ihren Perspektiven in Deliberationsprozesse einbezogen werden, ${ }^{3}$ wurde eine Vielzahl von Formaten entwickelt (u. a. Goldschmidt, 2014).

In der folgenden Darstellung soll das Potenzial einer spezifischen Variante eines Beteiligungsverfahrens für die transparente Deliberation und Partizipation von Bürger*innen aufgezeigt werden. Das „Bürgerdelphi Keimbahntherapie“ legten wir explorativ und ergebnisoffen an: Ziel war es, ein komplexes Thema für Lai*innen zu erschließen, sie zu einem informierten Urteil zu befähigen und „zu Resultaten zu gelangen, die an den aktuellen politischen Diskurs anschlussfähig sind“ (Grötker/Klatt, 2018: 10). Eines unserer Ziele war dabei auch, das Bürgerdelphi zu evaluieren, weswegen wir parallel eine Begleitforschung einrichteten (Cacean, 2019).

\subsection{Ziel und Methoden von BUEDEKA}

In einem Delphiverfahren werden Expert*innen zu einem auf unsicherem Wissen beruhenden, umstrittenen oder politisch unentschiedenen Sachverhalt befragt. In unterschiedlich ausgestaltbaren Prozessen wird die treffendste Beurteilung oder auch die Mehrheitsmeinung herausgefiltert (vgl. Häder, 2009). Das Bürgerdelphi verbindet diese Prinzipien mit Methoden eines Bürger*innenforums oder einer „Planungszelle“, durch die Bürger*innen über mehrere Monate und begleitet von Expert*innen und Moderation informierte und reflektierte Meinungen bezüglich einer gesellschaftlichen Problemlage ausbilden. Die Ergebnisse können dabei von unterschiedlicher Art sein: Sie können Lösungsvorschläge beinhalten oder aus der Zusammenstellung wichtiger Interessenskonflikte bestehen.

Für unser Bürgerdelphi wurden über Onlinenetzwerke, Anzeigen und Aushänge 26 Teilnehmende aus dem Raum Berlin gewonnen (18 Frauen, 9 Männer): Durchschnittsalter 42 Jahre, 18 mit Hochschulabschluss, davon 7 mit Promotion. Leider ergaben unse-

3 Zu Verständnis, Formen und Stellenwert von Partizipation siehe auch Winkler/Prainsack, Kap. 17, und Schicktanz/Buhr, Kap. 18. 
re Rekrutierungswege überwiegend Rücklauf aus einem akademischen Milieu, was die Teilnehmenden deutlich kritisierten. Das Bürgerdelphi gliederte sich in drei Phasen: Einer Vor-Ort-Auftaktveranstaltung am 28.04.2018 in Berlin, der „Remote“-Diskussionsphase von Mai bis Anfang Juli via Telefon und Onlineplattform, der Erstellung und Versendung des Abschlussberichts im September sowie der Abschlussveranstaltung im November mit einer Podiumsdiskussion im Rahmen der Berlin Science Week. ${ }^{4}$

Die halbtägige Auftaktveranstaltung hatte insbesondere zum Ziel, die Teilnehmenden in die Lage zu versetzen, als Gruppe Leitfragen zu entwickeln und sich hierfür Informationen und Anregungen von Expert*innenseite zu holen. Die Projektleitenden moderierten diesen Prozess der Fragenentwicklung, verdichteten die Ergebnisse und gaben Hilfestellung, wenn Debatten sich festfuhren. Gewappnet mit ihren eigenen Fragen traten die Teilnehmenden in Austausch mit einem Experten, Sascha Karberg, Wissenschaftsjournalist mit Schwerpunkt Life Sciences und Leiter der Wissenschaftsredaktion beim Berliner Tagesspiegel. Wir drehten also den gängigen Ablauf - erst Input, dann Diskussion - um, um den Teilnehmenden größtmögliche Souveränität über ihren Erkenntnisprozess zu geben. Auch wählten wir mit Sascha Karberg einen Vermittler mit Expertenstatus im Bereich Keimbahntherapie, der als Wissenschaftsjournalist an der Schnittstelle zwischen Forschung auf der einen Seite und Fragen und Anforderungen diverser Öffentlichkeiten auf der anderen Seite tätig ist. So hatten die Teilnehmenden einen Ansprechpartner, der sowohl die naturwissenschaftlichen Hintergründe als auch ihre gesellschaftlichen Implikationen im Blick hatte.

Nach dem Austausch mit Karberg arbeiteten die Teilnehmenden zentrale Leitfragen für das Bürgerdelphi heraus, die dann wiederum mit dem Experten diskutiert und dabei präzisiert wurden. Auch hier fungierten die Projektleitenden nur als Moderator*innen. Diese drei zentralen Fragen bildeten die Grundlage für die Diskussionsphase:

1. Sollte/dürfte die Keimbahntherapie eingesetzt werden, obwohl man die Folgen nicht kennt?

2. Kann ein Missbrauch der Keimbahntherapie effektiv verhindert werden?

3. Lässt sich die Keimbahntherapie wirklich regulieren? Und wenn ja, von wem?

Aus den drei Fragestellungen entwickelte das Projektleitungsteam Stellungnahmen, die in Form von Pro-Kontra-Positionen aufbereitet und den Teilnehmenden für die Diskussionsphase zur Verfügung gestellt wurden (eigene Recherchen waren in dieser „Remo-

4 Siehe unter: https://berlinscienceweek.com/about-science-week/ [16.06.2021]. 
te“-Phase selbstverständlich immer möglich). ${ }^{5}$ Die Interaktion der Projektleitung mit den Teilnehmenden sowie der Teilnehmenden untereinander erfolgte über individuelle Telefongespräche, schriftliche Befragungen sowie über eine Onlineplattform. Um die Kommunikationswege in der Begleitforschung vergleichen zu können, kommunizierte eine Gruppe nur über die Onlineplattform, die andere über Telefon und Plattform. Die Ergebnisse der Befragungen und Debatten wurden auf dem Onlineportal zusammengefasst. Aus dem Gesamtergebnis wurde ein Abschlussbericht (Grötker/Klatt, 2018) erstellt, der an ausgewählte Politiker*innen in Bundes- und Landespolitik, Gremien und Medien versandt wurde. Er war Diskussionsgegenstand mit Politiker*innen und Teilnehmenden bei der sehr gut besuchten Abschlussveranstaltung im Rahmen der Berlin Science Week und fand Niederschlag in einigen Medien. ${ }^{6}$

\subsection{Ergebnisse des Bürgerdelphis}

Wie nicht anders zu erwarten, bildete dieses Bürgerdelphi bei vielen Fragestellungen ein breites Meinungsspektrum ab. In einigen Punkten kamen die Teilnehmenden aber zu mehrheitlich vertretenen Ergebnissen. Insbesondere sprachen sie sich überwiegend dafür aus, das bestehende Verbot der Grundlagenforschung zur Keimbahntherapie in Deutschland zu lockern, weil sie die Ansicht vertraten, dass Deutschland damit eine aktive Rolle bei der internationalen Regulierung der Keimbahntherapie einnehmen kann. Damit kamen die Delphibeteiligten zu einer ganz ähnlichen Einschätzung wie der Deutsche Ethikrat (2019). Für diesen Standpunkt kann exemplarisch folgende Aussage stehen: „Wenn Deutschland sich hier nicht beteiligt, können wir international auch in Sachen Regulierung nicht mitreden und mitgestalten" (Grötker/Klatt, 2018: 49). Im Einzelnen erarbeiteten die Teilnehmenden zu allen drei Fragen Ergebnisse: Frage 1 bezog sich insbesondere auf die Chancen und Risiken einer Keimbahntherapie. Hier neigten die Teilnehmenden tendenziell der Einschätzung zu, einer an sich als risikobehaftet beurteilten Therapie doch Möglichkeiten der Umsetzung einzuräumen und diese durch weitere Forschung zu flankieren. Die Aussage eines/einer Teilnehmer*in illustriert ein Argumentationsmuster, das den Unterschied zwischen einem Eingriff in Körperzellen (= nicht vererbbar, sog. somatische Gentherapie $)^{7}$ und einem in Keimzellen (= vererbbar,

5 Das gesamte Material findet sich im digitalen Anhang zum Ergebnisbericht (Grötker/Klatt, 2018); siehe unter: http://www.explorat.de/wp-content/uploads/2018/BuedekaDigitalerAnhang.pdf [16.06.2021].

6 Siehe unter: http://www.buedeka.de/ [16.06.2021].

7 Siehe Fehse, Kap. 6, zum aktuellen Sachstand. 
sog. Keimbahntherapie) thematisiert. Er/sie stellt damit einen möglichen Nutzen der Erforschung der Keimbahntherapie für die somatische Gentherapie heraus:

Es gibt die Meinung, dass die somatische Gentherapie viel größere Chancen birgt als die Keimbahntherapie und dass von der somatischen Gentherapie auch mehr Menschen profitieren. Gerade die Forschung in Richtung Keimbahntherapie könnte aber auch helfen, genau jene biologischen Vorgänge besser zu verstehen, die es für die Weiterentwicklung der somatischen Gentherapie braucht. (Grötker/Klatt, 2018: 25)

Beim Thema "gesellschaftliche Risiken“ wurde von vielen Teilnehmenden die Gefahr eines Missbrauchs der CRISPR/Cas9-Technologie ebenso wie der Keimbahntherapie thematisiert (Grötker/Klatt, 2018: 28/30). Dabei wurde teilweise zwischen Grundlagenforschung, klinischer Forschung und Therapie nicht mehr differenziert. In den Äußerungen zeigte sich sogar Misstrauen insbesondere gegenüber der Forschung. Trotzdem leiteten die Teilnehmenden daraus kein Forschungsverbot ab, sondern plädierten überwiegend für mehr Forschung, um gesundheitliche Risiken zu minimieren. Der anscheinende Widerspruch löst sich vermutlich auf, wenn man bedenkt, dass es durchaus ein Misstrauen gegen die Forschung als Institution und gleichzeitig Vertrauen in einzelne Forschungsaktivitäten geben kann (und umgekehrt).

Im Fragenblock 3 sprachen sich die Teilnehmenden deutlich dafür aus, Grundlagenforschung zu ermöglichen, auch, um perspektivisch Erbkrankheiten wie die Mukoviszidose zu heilen. Das Argumentationsmuster, einer risikobehafteten Therapie nicht von vornherein das Vertrauen zu entziehen (auch mit Hinweisen auf historische Erfahrungen, etwa mit den Röntgenstrahlen), findet sich öfter. Gegen klinische Studien wandten sich die Teilnehmenden nicht, forderten also auch kein Moratorium. Sie befürworteten aber, dass die Durchführenden eine Selbstverpflichtung in Form eines Anforderungskatalogs eingehen. „Die Einigung auf eine Liste mit Anforderungen scheint international noch am ehesten machbar zu sein und am besten kontrollierbar" (Grötker/Klatt, 2018: 52).

Unsere Begleitforschung untersuchte unter anderem mithilfe der Argumentanalyse (Betz/Brun, 2016), wie sich die Argumentqualität entwickelte. Sie ergab den vorsichtigen Befund, dass sich über die verschiedenen argumentativen Runden eine leichte Steigerung der argumentativen Kompetenz messen ließ. Dabei unterschieden sich die beiden Gruppen (Telefongruppe, Digitalgruppe) nicht. Zudem untersuchten wir, ob das Projekt die üblichen Qualitätskriterien für Beteiligungsprojekte erfüllt (Kompetenzentwicklung, Effizienz, Fairness, Transparenz, Legitimität). Hierzu wurden die Teilnehmenden während der Auftaktveranstaltung sowie während und nach der Diskussions- 
phase befragt. Es zeigte sich, dass das Verfahren überwiegend als sehr gut bewertet wurde. Vielfach hörten wir: „Das könnte gerne öfter gemacht werden“, was angesichts des immensen Zeiteinsatzes der Teilnehmenden durchaus überraschte. Dieser Zeiteinsatz wurde dann auch teilweise kritisiert. Dennoch ist die Effizienz des Projekts als zufriedenstellend zu bewerten, insbesondere, da ein Teil der Arbeit „remote“, also online und/oder telefonisch, geleistet wurde, sodass aufwendige Treffen wegfielen (ein Aspekt, der in Pandemiezeiten besonders relevant für deliberative Prozesse ist). Die Teilnehmenden fühlten sich fair und transparent behandelt, was insbesondere für ihre Doppelrolle wichtig war: als Teilnehmende des Meinungsbildungs- und Beratungsprozesses und als Untersuchte in der Begleitforschung. Die Strategie, die Teilnehmenden vieles selbst erarbeiten zu lassen, wurde zwar als Form der Selbstwirksamkeit wertgeschätzt, führte allerdings auch zu Orientierungsschwierigkeiten. Ihren Abschlussbericht schätzten sie für die gesellschaftliche Debatte als relevant ein, einige sogar als „innovativ“ (Grötker/Klatt, 2018: 30). Wir konnten zeigen, dass ein „Remote“-Prozess in Kombination mit einer Auftaktveranstaltung, die mit geeigneten Methoden Verbindlichkeit herstellt (Cacean, 2019), umsetzbar ist und dadurch auch in größerem Umfang durchführbar wird. Allerdings steht und fällt ein solches Projekt mit der Qualität der Onlinepräsenz, was auch die Kosten in die Höhe treiben kann.

\subsection{Fazit}

Bürgerdelphis können sowohl im Rahmen einer partizipativen Politikberatung eingesetzt werden als auch, um qualifizierte Meinungsbildungsprozesse bei Bürger*innen anzustoßen und sie eigene fundierte Argumentationslinien rund um wissenschaftliche und ethisch relevante Sachverhalte finden zu lassen. Sie können also ein Element der Beteiligung und der qualifizierten Willensbildung sein. In unserem Bürgerdelphi arbeiteten die Teilnehmenden zentrale ethische Fragen zur Keimbahntherapie mittels CRISPR/Cas heraus, die ihren Alltag, ihre Lebensentscheidungen und das Wohl und Wehe zukünftiger Generationen berühren - und kamen dabei unabhängig teilweise zu ähnlichen Einschätzungen wie der Deutsche Ethikrat. Kurz nach Beendigung unseres Bürgerdelphis trat genau der Ernstfall ein, auf den sich die Teilnehmenden in gewisser Weise vorbereitet hatten: die intensive Debatte, die auf den Eingriff in die Keimbahn der chinesischen Zwillinge durch den chinesischen Forscher He folgte. Im Sinne des Deliberationsgedankens hatten unsere Teilnehmenden vorab Sach- und Argumentationskompetenz erworben, die sie vermutlich gut auf diese gesellschaftliche Debatte vorbereitet haben. Wie stark das Verfahren durch Anschlusskommunikation in die Familien und Netzwerke wirkte, können wir nur vermuten; das sollte in zukünftigen Verfah- 
ren in die Beobachtung einbezogen werden. Durch Verkopplung mit einer Lehrveranstaltung am Karlsruher Institut für Technologie (KIT) hat das Verfahren den wissenschaftlichen Nachwuchs erreicht. Wie stark ein Empowerment durch ein Bürgerdelphi fortwirkt, wäre eine anschließende Untersuchung wert. Bürgerdelphis sind kleinteilige Verfahren, sodass der Aufwand gut im Blick behalten werden muss. Hier kann die geschickte Verknüpfung von Vor-Ort-Arbeit mit Arbeit auf Onlineplattformen helfen, welche allerdings hervorragende Schnittstellen für die Interaktion bieten müssen. Um den Teilnehmendenkreis über die Welt der Universitätsabsolvent*innen hinaus zu öffnen - die sich auf unsere Aufrufe überwiegend gemeldet hatten -, empfiehlt sich eine aufsuchende Strategie, um etwa in Berufsschulen für die Teilnahme zu werben (Humm et al., 2020).

\subsection{Literaturverzeichnis}

Berkeley News (2018): CRISPR co-inventor responds to claim of first genetically edited babies. OnlinePublikation 26.11.2018. Unter: https://news.berkeley.edu/2018/11/26/doudna-responds-to-claimof-first-crispr-edited-babies/ [14.06.2021].

Betz, G./Brun, G. (2016): Analysing practical argumentation. In: Hanson, S. O./ Hirsch Hadorn, G. (Hrsg.): The argumentative turn in policy analysis. Reasoning about uncertainty. Springer, Dordrecht: 39-77.

Cacean, S. (2019): Forschungsbericht Bürgerdelphi Keimbahntherapie. Karlsruher Institut für Technologie. DOI: 10.5445/IR/1000096788.

Deutscher Ethikrat (2018): Anwendung von Keimbahneingriffen derzeit ethisch nicht vertretbar. Unter: https://www.ethikrat.org/mitteilungen/mitteilungen/2018/anwendung-vonkeimbahneingriffen-derzeit-ethisch-nicht-vertretbar/\#: :text=Die\%20am\%20heutigen\%20 Montag\%2C\%20dem,ernste\%20Verletzung\%20ethischer\%20Verpflichtungen\%20dar. [14.06.2021].

Deutscher Ethikrat (2019): Stellungnahme: Eingriffe in Die Menschliche Keimbahn. Unter: https:// www.ethikrat.org/mitteilungen/2019/ethikrat-keimbahneingriffe-derzeit-zu-risikoreich-aberethisch-nicht-grundsaetzlich-auszuschliessen/ [14.06.2021].

Goldschmidt, R. (2014): Kriterien zur Evaluation von Dialog- und Beteiligungsverfahren. VS Verlag für Sozialwissenschaften, Wiesbaden.

Grötker, R. (2017): Expertenkonsultationen und Stakeholder-Befragungen mit Deliberativem Delphi. (Expert consultations and stakeholder-surveys with Deliberative Delphi). Unter: https://papers. ssrn.com/sol3/papers.cfm?abstract_id=3256258 [14.06.2021].

Grötker, R./Klatt, K. (2018): Ergebnisbericht Bürgerdelphi Keimbahntherapie. Karlsruher Institut für Technologie. DOI: 10.5445/IR/1000096789. 
Häder, M. (2009): Delphi-Befragungen: Ein Arbeitsbuch. Lehrbuch. VS Verlag für Sozialwissenschaften, Wiesbaden.

Humm, C. et al. (2020): Feeling left out: Underserved audiences in science communication. In: Media and Communication 8(1): 164-176.

Normile, D. (2018): CRISPR bombshell: Chinese researcher claims to have created gene-edited twins. Online-Publikation 26.11.2018. Unter: https://www.sciencemag.org/news/2018/11/crispr-bombshell-chinese-researcher-claims-have-created-gene-edited-twins [14.06.2021]. 